\title{
Self-Reported Oral Hygiene Practices and Periodontal Status of Visually Impaired Adults
}

\author{
Tuti Ningseh Mohd-Dom (Corresponding author) \\ Department of Dental Public Health, Faculty of Dentistry, Universiti Kebangsaan Malaysia \\ Jalan Raja Muda Abdul Aziz, 50300 Kuala Lumpur, Malaysia \\ Tel: 603-9289-7731 Email: tutinin@gmail.com \\ Rokiah Omar \\ Department of Optometry, Faculty of Allied Health Sciences, Universiti Kebangsaan Malaysia \\ Jalan Raja Muda Abdul Aziz, 50300 Kuala Lumpur, Malaysia \\ Nor Aida Abdul Malik \\ Faculty of Dentistry, Universiti Kebangsaan Malaysia \\ Jalan Raja Muda Abdul Aziz, 50300 Kuala Lumpur, Malaysia \\ Khairunnisa Saiman \\ Faculty of Dentistry, Universiti Kebangsaan Malaysia \\ Jalan Raja Muda Abdul Aziz, 50300 Kuala Lumpur, Malaysia \\ Nu'amirazura Rahmat \\ Department of Optometry, Faculty of Allied Health Sciences, Universiti Kebangsaan Malaysia \\ Jalan Raja Muda Abdul Aziz, 50300 Kuala Lumpur, Malaysia
}

\begin{abstract}
This study investigated self-reported oral hygiene practices and periodontal status of visually impaired adults. A convenient sample of visually impaired adults who were trainees at the Malaysian Association for the Blind (MAB) participated in the study. Data were collected through a face-to-face interview and clinical dental examination. An assessment of visual acuity determined types of visual impairment. Thirty-nine adults were examined (response rate $=65.0 \%$ ). Most were females $(56.4 \%)$ and aged between 21-30 years $(66.7 \%)$. About half were blind, $20.5 \%$ had low vision and the rest had at least one eye with normal vision. Many reported to brush teeth at least twice a day $(82.1 \%)$, used toothbrush and toothpaste $(97.4 \%)$ but hardly used dental floss. Almost all had experienced symptoms like tooth sensitivity (53.8\%) and inflamed and painful gums (43.6\%). Average percentage of sites with plaque and bleeding were $81.7 \%$, and 35.1 respectively. Although good oral hygiene practices were reported, periodontal status was poor and periodontal treatment needs were high.
\end{abstract}

Keywords: Oral hygiene, Dental awareness, Visually impaired

\section{Introduction}

"Just because a man lacks the use of his eyes doesn't mean he lacks vision."

-Stevie Wonder-

Visual impairment is a problem with one's ability to see and can range from low vision to total blindness. In Malaysia, the prevalence of people with visual impairment has been estimated to be about $2.42 \%$ for low vision and $0.28 \%$ for total blindness (Zainal et al 2002). Of people with all types of disabilities, this group account for about $9.5 \%$ (Welfare Department of Malaysia 2005). Although the prevalence is not so high, oral diseases can greatly affect their quality of life and would in turn worsen their capability to access oral health care. These 
visually impaired patients present a unique population that challenges the dentist's skills and knowledge. They differ from normal patients with regard to the professional relationship between patient and the dentist. In addition, the true ability and talents of the blind are often underestimated. Providing comprehensive dental care for the visually impaired is not only rewarding but is also a community service that health care providers should fulfill. These patients may be managed well when the oral health care provider undertakes adequate training and understanding of the needs of individuals with low visions.

People with visual impairment are at an increased risk of developing oral diseases, namely periodontal disease because of greater difficulty in attaining good oral hygiene. This may be due to the lack of ability to visually assess whether dental plaque has been effectively removed or if their gums bleed during tooth brushing. Often, dental plaque is an important prerequisite for the development of dental caries and periodontal diseases. People with low vision cannot detect and recognize early signs of oral disease and because of this they may not be able to take the necessary action to prevent or treat a particular oral tcondition. A study among visually impaired adolescents (Azrina et al 2007) observed that difficulties faced during tooth brushing include putting toothpaste on brush and using brushing techniques that could be detrimental to oral structures; such as brushing using hard bristles and using strokes that damage the periodontal ligaments. Other than skills in plaque control, factors that have been suggested to affect oral health of the general population include lack of dental knowledge and regular visits to the dentist. The same could be said for the visually impaired population.

There is some evidence in the published literature which highlighted the need to provide more oral health education for people with visual impairment (Azrina et al 2007, O'Donnell \& Crosswaite 1990, Yalcinkaya \& Atalay 2006). This recommendation appeared to have been based on the notion that knowledge of dental health is important to promote the maintenance of oral health. A Taiwan study, had established that knowledge of dental health among visually impaired students were poor compared to their sighted peers (Chang \& Shih 2004). The same study also found that visually impaired students were less likely to practice good oral health habits.

In Malaysia, although there is huge success with regard to the school dental service, there has not been much information on its impact on the visually impaired school children. Furthermore, not much is known about this group after they have left school. Even among the general population, it is known (Ministry of Health, 2006) that oral health does deteriorate once people leave school and reach adulthood. The same or worse could be expected within these populations with visual impairment.

This study aims to provide an insight into self-reported oral hygiene practices, oral health knowledge and periodontal status of adults who are visually impaired. If any oral health intervention were to be carried out, the need for baseline data is crucial to provide the input on which aspects of oral health promotion and services that need to be improved.

\section{Methodology}

\subsection{Sample}

The participants were trainees at the Malaysian Association for the Blind (MAB), a premier voluntary organization in Malaysia that looks after the general well-being of blind persons in the country. All participants had some form of visual impairment and did not have any other disabilities or impairments such as loss of limbs, impaired speech or hearing. All 60 trainees were included in the sampling frame and were invited to participate in this cross-sectional study involving a face-to-face interview, an oral examination and assessment of visual impairment. They were scheduled for the data collection session after formal classes ended for the day.

\subsection{Face-to-face interview}

The interview was conducted by interviewers who had been briefed prior to the data collection. It was done using a pre-tested, structured questionnaire form to collect the following: personal information, oral hygiene habits, dental service utilization and dental knowledge. The section on dental knowledge included eight close-ended questions knowledge related to causes, prevention, signs and treatment of dental caries and knowledge related to causes, prevention, signs and treatment of periodontal disease.

\subsection{Dental examination}

The status of periodontal health provides an indication of oral hygiene status when assessed using Modified Visible Plaque Index (VPI) and modified Gingival Bleeding Index (GBI). The data for VPI were obtained by visual detection while for GBI, it was obtained by running the periodontal probe along the gingival margin and the presence of bleeding was observed. Buccal and lingual surfaces of all teeth were examined for plaque and bleeding sites using both indices. Sites with plaque or bleeding were recorded as "1" while those without were recorded as "0". All teeth were charted except for retained root or missing teeth which were charted as "X" - 
meaning not applicable.

To estimate the needs for periodontal treatment, the Community Periodontal Index for Treatment Needs (CPITN) - with some modifications in relation to treatment needs - was used. This index was introduced by the World Health Organisation (WHO 1997) and recommended to be used as a screening tool so that countries can plan effective intervention programmes for the prevention and control of periodontal disease. This involved charting of six index teeth, each representing a sextant of the dentition. The standard parameters for the presentation of data are percentage of persons by their highest CPI score (prevalence rate) and the mean number of sextants (severity) with certain CPI scores: score $0=$ healthy periodontal conditions, score $1=$ gingival bleeding, score $2=$ gingival bleeding and dental calculus, score $3=$ shallow periodontal pockets $(4-5 \mathrm{~mm})$, score $4=$ deep periodontal pockets $(6 \mathrm{~mm}$ or more), score $9=$ excluded, and score $X=$ not recorded or not visible. Presence of plaque, gingival bleeding and dental calculus are also indications of oral hygiene status.

The dental examination was carried out in a hall setting by three examiners who took turns to examine. Prior to the data collection period, these examiners underwent a standardization session for all three indices and were calibrated with a periodontist who acted as the gold standard. However, during the actual data collection, it was not possible to carry out a duplicate examination on the study respondents due to time constraint.

\subsection{Assessment of visual acuity}

Visual impairment was assessed by the optometry team using distant visual acuity (VA) measurement. VA is an indication of the clarity or clearness of one's vision and often measured according to the size of letters viewed on a chart. VA is expressed relative to 20/20 if using "foot" as a unit of measurement, or 6/6 if using the metre. In this study LogMAR or Minimal Angle of Resolution charts are used to measure distant VA. Subjects were asked to stand $3 \mathrm{~m}$ away from the charts to measure habitual VA. To obtain distant VA for the right eye, the left eye was covered, and vice versa. For participants who had difficulty seeing the topmost line, (1.0LogMAR) charts, they were asked to move forward for a specified distance of $2 \mathrm{~m}$ and $1 \mathrm{~m}$. If they were still not able to see the line, they were then assessed whether they were able to count number of fingers shown from a distance of $50 \mathrm{~m}(\mathrm{CF})$, able to distinguish whether a hand is moving or not (HM) or able to perceive presence of light. Not being able to perceive light will be interpreted as total blindness.

\subsection{Data processing and analysis}

The data collected were entered and analysed using SPSS version 15. Responses to most of the questionnaire items were reported as percentages. The VPI and GBI scores were calculated as the percentage of sites with plaque or bleeding over the total number of sites charted. Mean percentages for different regions anterior/posterior, upper/lower, buccal/lingual - were reported separately. CPITN scores were reported as the worst score among all six scores from the sextants. As for visual acuity assessment, the vision is determined using the reading from the better eye and reported as percentages of participants at a particular level of visual impairment.

\section{Results}

A total of 39 adults took part in the study (response rate $=65.0 \%)$. Majority of subjects were females $(\mathrm{n}=22$, $56.4 \%)$ and aged between 21-30 years $(n=19,66.7 \%)$. About half $(n=20,51.3 \%)$ of the trainees were blind, $20.5 \%(\mathrm{n}=8)$ had low vision and the rest $(\mathrm{n}=11,28.2 \%)$ had at least one eye with normal vision.

\subsection{Oral hygiene practices}

$17.9 \%$ reported to brush only once a day, yet up to $43.6 \%$ claimed to brush more than twice a day. Use of toothbrush and toothpaste was high $(97.4 \%)$, while dental floss use was low $(10.3 \%)$. Other oral health aids used are mouthrinses $(17.9 \%)$ and toothpicks (15.4\%). Majority reported dental attendance only when there is need for treatment $(69.2 \%)$, while some (20.5\%) cited no experience of dental treatment. About half $(53.8 \%)$ reported a dental visit in more than twelve months before. Reasons for last dental treatment were for dental check-up /scaling (35.9\%), restorative (12.8\%), emergency (23.1\%) and other reasons $(15.4 \%)$.

\subsection{Knowledge on dental caries}

Most respondents knew that bacteria in the mouth (92.3\%) and sugary food (94.9\%) were factors that can cause dental caries. Most (97.4\%) perceived that good oral hygiene care can prevent dental caries and this was consistent with $84.6 \%$ agreeing that brushing teeth after meals and reducing sugary food $(92.3 \%)$ can prevent the dental caries. A similar percentage which is $82.1 \%$ of the subjects, agree that gargling after meal and usage of fluoridated toothpaste can prevent the dental caries. Many (79.5\%) of them agreed that regular dental check-up can also lead to caries prevention. Majority of the trainees identified cavities $(92.3 \%)$, toothache $(82.1 \%)$, oral 
malodour $(74.4 \%)$ and loose teeth $(74.4 \%)$ as symptoms of dental caries. Many knew that restoration $(84.6 \%)$ is treatment for dental caries, followed by $79.5 \%$ for extraction. Some $(38.5 \%)$ agreed that taking painkiller can treat dental caries, whereas few $(5.1 \%)$ perceived that if you ignore it, dental caries can be treated on its own.

\subsection{Knowledge on periodontal disease}

Similar response (87.2\%) was cited for two factors causing periodontal disease - which are bacteria in the mouth and poor oral hygiene. This is followed by injury while brushing (76.9\%), while calculus and sugary food was agreed by $71.8 \%$ of the participants, followed by smoking (59.0\%) and systemic diseases (48.7\%). All patients recognised "inflamed and pain gingival" as symptoms of periodontal disease. Other symptoms identified were "gingival bleeding while tooth brushing" (92.3\%), tooth sensitivity $(79.5 \%)$, loose tooth $(74.4 \%)$ and oral maolodour (69.2\%). Good oral hygiene care and regular check-up were cited by almost all respondents (97.4\%) as factors that can prevent periodontal disease. This was followed by reduction of sugary food $(84.6 \%)$ and brushing teeth after meal (74.4\%). Almost half of the participants (48.7\%) perceived that antibiotic / medication could treat periodontal disease followed by cleaning and removing all plaque and calculus (38.5\%). $12.8 \%$ of the subjects admitted that they did not know the treatment for periodontal disease.

\subsection{Previous experience of oral symptoms}

More than half of the respondents $(58.3 \%)$ disclosed that they had experienced tooth sensitivity, followed by inflamed and pain gingival experience $(43.6 \%)$ and oral malodour $(30.8 \%)$. About one-quarter $(28.2 \%)$ of the respondents reported having loose teeth and gingival bleeding while tooth brushing. Needs for scaling (35.9\%) were the most perceived, followed by periodontal treatment (23.1\%), restoration (17.9\%), extraction (10.3\%) and other types of treatment $(12.8 \%)$.

\subsection{Periodontal status}

The average percentage of sites with dental plaque was $81.7 \%$ (Table 1), with anterior segments scoring better than the posterior ones. Posterior lower lingual segment appears to have the highest mean percentage of sites with plaque $(90.1 \%)$. The average percentage of bleeding sites was $35.1 \%$ (Table 2) with similar distribution as plaque - anterior segments were healthier and the highest mean percentage was also on the posterior lingual teeth (49.5\%). As high as $69.2 \%$ of participants scored 2 (dental calculus) as the highest CPI score (Table 3). This corresponded with the proportion for highest treatment need for simple scaling (Table 4).

\subsection{Relationship between periodontal disease and vision}

Plaque levels were seen to be highest among the normal-vision group and lowest among the blind (Table 5). These differences were not found to be statistically significant. On the other hand, scores for gingival bleeding were found to be highest among the blind, followed by the normal-vision group (Table 6). These differences were demonstrated to be statistically significant (Kruskall-Wallis Test, $\mathrm{p}<0.01$ ).

\section{Discussion}

This study is limited by the moderate response rate, small sample size, and timing of the data collection which was late in the day and did not permit duplicate of the clinical examination. Furthermore, the questionnaire used had been developed for sighted populations and may have validity and reliability issues. However, its findings can be seen to confirm that in this particular target group, poor oral hygiene, high levels of periodontal disease and unmet treatment need was evident (Azrina et al 2007, Anaise 1979, Mahoney et al 2008, Chang \& Shih 2004).

Basic oral health knowledge was moderate as evidenced by many respondents getting the answers right for etiology, prevention, symptoms and treatment for both caries and periodontal disease. However, there were some concerns over misconceptions (for example: sugary food can cause periodontal disease; which is not true) and lack of exposure in certain areas (for example: the role of smoking in periodontal disease.)

Reported oral hygiene behavior was acceptable for frequency of brushing although, the percentage reporting brushing once a day was higher than the normal population as opposed to the recommended at least twice a day (Ministry of Health, 2006). Low use of dental floss was similar to that of the general population, while use of mouthrinses was higher in this study (Ministry of Health 2006) Utilization behavior appeared to be inadequate, with high reports of symptomatic attendance, and even evidence of non-attendance. Another interesting finding is that more than half of the respondents admitted having experienced a dental symptom - namely; tooth sensitivity, gingivitis and oral malodour. It is unfortunate that in spite of having these symptoms, most of them have not visited the dentist for a long time.

The question of access needs to be explored to identify barriers to care so that an effective oral health 
intervention may be planned and executed (Nandini 2003, Yalcinkaya \& Atalay 2006). There is a possibility that most of the people would only seek dental treatment if they were in great pain, otherwise they would think that their oral condition was good even it was not; especially because they lack the capacity to detect signs of abnormalities within the oral cavity in a visual manner. Moreover, some would just bear the pain hoping that it would resolve.

Two of the most common oral diseases affecting mankind are dental caries and periodontal disease. Although the disease mechanisms are different both diseases are initiated by the presence of microbial dental plaque. Dental plaque that is present on tooth surfaces will affect the oral hygiene status of an individual. If it is not removed from the tooth surface through effective oral hygiene measures, it will lead to inflammation of the gingival tissues, also known as gingivitis which is characterized among others as bleeding of the gingival tissues upon probing. Gingivitis is also considered as the mildest form of periodontal disease and one that is reversible. Subsequently dental plaque will undergo calcification, harden and form dental calculus. So in other words, presence of dental plaque, gingival bleeding and dental calculus are indications of oral hygiene status and this directly affects periodontal status. At a more advanced level of periodontal disease, chronic periodontitis can develop; and this is defined as an infectious disease resulting in inflammation within the supporting tissues of the teeth, progressive attachment, and bone loss (American Academy of Periodontology 2001).

In this study, the overall percentage of sites with plaque for all the subjects was a mean of $81.7 \%$. This can be considered as poor oral hygiene due to ineffective plaque control or oral hygiene measures. However, the absence of a control group measuring plaque levels makes it difficult to conclude that plaque levels among the low vision group is worse off compared to a sighted population. What may be concluded is that plaque level for this group was high and it should be of concern as this may lead to dental caries or periodontal disease. The percentage of sites that had gingival bleeding was low to moderate and this implies that the extent of active periodontal disease was not severe in this study.

In the most recent nationwide survey in Malaysia, which was the National Oral Health Survey of Adults (NOHSA) conducted in 2000 (Oral Health Division 2004), it was found that for the 15-19 years age group, $25.8 \%$ had healthy periodontium. The remaining had varying degrees of periodontal disease including gingivitis $(11.2 \%)$, dental calculus $(60 \%)$, shallow periodontal pockets of $4-5 \mathrm{~mm}$ depths $(2.9 \%)$ and deep pockets of $6 \mathrm{~mm}$ and more $(0.1 \%)$. In this study, only $2.6 \%$ had healthy periodontium, $10.3 \%$ with gingivitis, $69.2 \%$ with calculus, $10.3 \%$ with shallow pockets and $7.7 \%$ with deep pockets. In comparison with the general population, there was a smaller proportion of individuals in this study who have healthy periodontium yet a much higher proportion with the most advanced periodontal condition. This indicates that many had poor oral hygiene which would require dental scaling procedures to clean the affected tooth surfaces and some actually need complex specialist care.

Among the three groups of vision classification: normal (one eye normal), low vision and the blind, plaque levels were highest for the normal vision group, but this difference was not found to be statistically significant. On the other hand, scores for gingival bleeding were found to be highest among the blind (Kruskall-Wallis Test, $\mathrm{p}<0.01$ ). Gingivitis is an inflammatory process affecting the soft tissues surrounding the teeth, the most common type is one where bacteria is its primary cause (plaque-related) which colonises the gingival sulcus and adjacent tooth surfaces. This relationship between plaque deposits as one that is of cause-and-effect has long been established (Anerud et al 1979, Loe et al 1986) and thus demonstrates that bleeding of the gingiva is a sequelae of prolonged plaque accumulation and retention on tooth surfaces. So although, plaque levels appear to be the same among the three types of vision classification, active disease is highest among the blind.

Recommendations for future research include ensuring a larger sample size to allow for a better representation of the target population and to improve external validity of results in future study. It is highly desirable that questionnaires are developed specifically for the blind and translated into Braille to improve internal validity of the study. Qualitative research methods to be employed for interviews and various observation methods can provide an insight into more in-depth information and greater wealth of data. The understanding of the capabilities and limitations of those impaired with visual-related conditions will help the dental care providers to facilitate quality care. Moreover, only with keen collaborations with other members of healthcare team can comprehensive health care be achieved.

\section{Conclusions}

Oral hygiene practice; which was self-reported was generally acceptable except that the proportion of brushing once a day was higher than the general population. Use of dental floss was low, similar to the general population. Most were symptomatic attendees while some reported that they never had any form of dental treatment. Oral health knowledge was moderate with some questions answered correctly but with some evidence of 
misconceptions and lack of exposure. Periodontal status was poorer than the general population with many requiring treatment as assessed normatively as well as reported by the respondents. Sites with bleeding on probing were found to be significantly highest among the blind group $(\mathrm{p}<0.05)$, while those with plaque show no significant differences between different visual groups.

\section{Acknowledgements}

Our thanks to the Management and the trainees at Malaysian Association for the Blind for without their support and cooperation, this study would not have been possible. Our appreciation also goes to Dr Shahida Mohd Said, Dr Huwaida Abd Halim, Dr Su Wei Siong, DSA Marlina Hassan and the whole of the optometry team whose role in the clinical examination was most instrumental.

\section{References}

American Academy of Periodontology. (2001). Position Paper: Guidelines for periodontal therapy. J Periodontol, 72(11), 1626-1628.

Anaise Z. J. (1979). Periodontal disease and oral hygiene in a group of blind and sighted Israeli teenagers (14- 17 years age). Comm Dent Oral Epidemiol, 7, 353-6.

Anerud, A., Loe, H., Boysen, H. \& Morrision, E. (1979). Natural history of periodontal disease in man: changes in gingival health and oral hygiene before 40 years of age. $J$ Periodontal Res, 14, 526-540.

Azrina, A. N, Norzuliza, G. \& Saub, R. (2007). Oral hygiene practices among the visually impaired adolescents. Annals Dent Univ Malaya, 14, 1- 6.

Loe, H. Anerud, A, Boysen, H. \& Morrison, E. (1986). Natural history of periodontal disease in man. Rapid, moderate, and no loss of attachment in Sri Lankan labourers 14-46 years of age. $J$ Clin Periodontol, 13, 431-445.

Mahoney, E.K., Kumar, N. \& Porter, S.R. (2008). Effect of visual impairment upon oral health care: a review. $\mathrm{Br}$ Dent J, 204(2), 63-7.

Ministry of Health, Malaysia. (2005). Health Management Information System Annual Report. Government Printer, Kuala Lumpur.

Nandini, N.S. (2003). New insights into improving the oral health of visually impaired children. $J$ Indian Soc Ped Prev Den, 2 21, 142-3.

O' Donnell, D. \& Crosswaite, M.A. (1990). Dental health education for the visually impaired child. $J$ R Soc Health, 110(2), 60-1.

Oral Health Division, Ministry of Health. (2004). National oral health survey of adults 2000. Kuala Lumpur.

Shih, Y.H. \& Chang, C.H.S. (2004). Knowledge of dental health and oral hygiene practices of Taiwanese visually impaired and sighted students. $J$ Visual Imp and Blindness, 98(5), 1-15.

Welfare Department of Malaysia. (2006). [Online] Available: http://www.jkm.gov.my/jkm (October, 2009).

World Health Organisation. (1997). Oral health surveys basic methods. $\left(4^{\text {th }} \mathrm{ed}\right)$, Geneva.

Yalcinkaya, S.E. and Atalay T. (2006). Improvement of oral health knowledge in a group of visually impaired students. Oral Health Prev Dent, 4, 243-53.

Zainal, M., Ismail, S.M., Ropilah, A.R. et al. (2002). Prevalence of blindness and low vision in Malaysian population: results from the National Eye Survey 1996. Br J Ophtalmol, 86, 951-6. 
Table 1. Average percentage of sites with plaque

\begin{tabular}{lc}
\hline Site & Mean percentage (S.D) \\
\hline Full mouth & $81.7(23.6)$ \\
Anterior upper buccal & $78.7(36.8)$ \\
Anterior upper palatal & $75.1(37.0)$ \\
Posterior upper buccal & $82.9(26.3)$ \\
Posterior upper palatal & $84.4(26.3)$ \\
Anterior lower buccal & $80.9(36.1)$ \\
Anterior lower lingual & $78.8(35.7)$ \\
Posterior lower buccal & $81.2(30.7)$ \\
Posterior lower lingual & $90.1(20.4)$ \\
\hline
\end{tabular}

Table 2. Average percentage of sites with bleeding on probing

\begin{tabular}{lc}
\hline Site & Mean percentage (S.D) \\
\hline Full mouth & $35.1(33.6)$ \\
Anterior upper buccal & $33.3(40.4)$ \\
Anterior upper palatal & $27.1(36.8)$ \\
Posterior upper buccal & $41.5(37.2)$ \\
Posterior upper palatal & $34.2(39.6)$ \\
Anterior lower buccal & $31.4(41.1)$ \\
Anterior lower lingual & $33.3(40.5)$ \\
Posterior lower buccal & $28.7(38.5)$ \\
Posterior lower lingual & $49.5(36.7)$ \\
\hline
\end{tabular}

Table 3. Distribution of subjects according to the highest CPI score

\begin{tabular}{lc}
\hline Score & Number $\mathbf{( \% )}$ \\
\hline Healthy & $1(2.6)$ \\
Bleeding & $4(10.3)$ \\
Calculus & $27(69.2)$ \\
Pocket $4-5 \mathrm{~mm}$ & $4(10.3)$ \\
Pocket $6 \mathrm{~mm}$ or more & $3(7.7)$ \\
\hline
\end{tabular}

Table 4. Distribution of subjects according to the highest score for periodontal treatment need

\begin{tabular}{lc}
\hline Score & Number $(\mathbf{\%})$ \\
\hline No treatment & $0(0.0)$ \\
Oral hygiene instruction & $5(12.8)$ \\
Simple scaling & $27(69.2)$ \\
Deep scaling & $4(10.3)$ \\
Complex treatment & $3(7.7)$ \\
\hline
\end{tabular}


Table 5. Distribution of sites with plaque by classification of vision

\begin{tabular}{lll}
\hline Classification of vision & $\begin{array}{l}\text { Mean percentage of sites with } \\
\text { plaque (S.D) }\end{array}$ & Mean rank of percentages $(*)$ \\
\hline Normal & $48.1(10.4)$ & 23.7 \\
Low & $44.8(16.6)$ & 22.5 \\
Blind & $40.5(13.3)$ & 17.0 \\
\hline
\end{tabular}

*Kruskall Wallis Test, $\mathrm{p}>0.05$

Table 6. Distribution of sites with gingival bleeding by classification of vision

\begin{tabular}{lll}
\hline Classification of vision & $\begin{array}{l}\text { Mean percentage of sites with } \\
\text { bleeding (S.D) }\end{array}$ & Mean rank of percentages $(*)$ \\
\hline Normal & $15.0(16.2)$ & 18.0 \\
Low & $4.0(5.2)$ & 8.4 \\
Blind & $26.2(17.9)$ & 25.8 \\
\hline
\end{tabular}

*Kruskall Wallis Test, $\mathrm{p}<0.05$ 